Теорія Ймовір. та Матем. Статист. Вип. 79, 2008
Theor. Probability and Math. Statist.

No. 79, 2009, Pages 117-126

0094-9000(09)00774-1

Article electronically published on December 28, 2009

\title{
PROPERTIES OF SOLUTIONS OF STOCHASTIC DIFFERENTIAL EQUATIONS WITH NONHOMOGENEOUS COEFFICIENTS AND NON-LIPSCHITZ DIFFUSION
}

UDC 519.21

\author{
YU. S. MISHURA, S. V. POSASHKOVA, AND G. M. SHEVCHENKO
}

\begin{abstract}
Properties of solutions of stochastic differential equations with nonhomogeneous coefficients and non-Lipschitz diffusion are studied in the paper. Conditions on the coefficients of an equation are obtained ensuring that a solution does not vanish over a finite time interval in the case of the diffusion $\sigma(t) \sqrt{x}$. We prove a limit theorem that solutions continuously depend on the parameter $n$ in the space $L_{1}(\mathrm{P})$ for a sequence of stochastic differential equations with nonhomogeneous coefficients and non-Lipschitz diffusion.
\end{abstract}

\section{INTRODUCTION}

The main aim of this paper is to analyze the behavior of solutions of stochastic differential equations with nonhomogeneous coefficients in the case of a diffusion that does not satisfy the Lipschitz condition. Stochastic differential equations of this kind are often used when modelling various processes in financial markets, in particular, primary and derivative prices, interest rates, etc.

A classical example of such an equation appearing in financial mathematics is the one related to the Cox-Ingersoll-Ross model describing the evolution of the interest rate; namely,

$$
r_{t}=r_{0}+\int_{0}^{t} a\left(b-r_{s}\right) d s+\int_{0}^{t} \sigma \sqrt{r_{s}} d W_{s}, \quad r_{0}>0
$$

where $a$ and $\sigma$ are real positive constants. If $r_{t}>b$, then the nonrandom drift factor is negative, while if $r_{t}<b$, then it is positive. Thus a path of a solution of this equation tends towards the mean value $b$. It is known (see, for example, [1]) that the equation has a unique solution and if $a b \geq \sigma^{2} / 2$, then this solution does not vanish with probability one.

The latter property is important for the financial models, since the processes modulating the prices of financial assets and interest rates have to be positive. The question on hitting or nonhitting of a certain domain (the negative semi-axis, in particular) is studied in several papers (see, for example, [1, Section VI]) in the case of homogeneous diffusion processes. However, many applications lead to equations whose coefficients are not homogeneous. These are the equations studied in this paper.

2000 Mathematics Subject Classification. Primary 60H10; Secondary 91B28.

Key words and phrases. Stochastic differential equations, non-Lipschitz diffusion, Cox-Ingersoll-Ross model, continuous dependence on a parameter. 
Some results concerning the existence, uniqueness, and continuity of trajectories of a solution of a stochastic differential equation with nonhomogeneous coefficients and non-Lipschitz diffusion are discussed in Section 2 below.

In Section 3] we find sufficient conditions that a solution does not vanish over a finite time interval in the case of a stochastic differential equation with nonhomogeneous coefficients and with the diffusion $\sigma(t) \sqrt{x}$. The result obtained in the paper can be applied to the Cox-Ingersoll-Ross model whose corresponding equation has coefficients $a, b$, and $\sigma$ depending on the time argument $t$. In particular, if $a(t) b(t)>\sigma^{2}(t) / 2$, then a solution does not vanish over a finite time interval.

In Section 4. we prove a limit theorem that solutions continuously depend on the parameter $n$ in the space $L_{1}(\mathrm{P})$ for a sequence of stochastic differential equations with nonhomogeneous coefficients and non-Lipschitz diffusion.

\section{Existence, Uniqueness, AND CONTINUity of trajeCtORIES OF A SOLUtion}

We consider the following stochastic differential equation:

$$
X(t)=X_{0}+\int_{0}^{t} b(s, X(s)) d s+\int_{0}^{t} \sigma(s, X(s)) d W(s), \quad t \geq 0,
$$

where the initial value $X_{0} \in \mathbf{R}$ is nonrandom, the coefficients $b: \mathbf{R}^{+} \times \mathbf{R} \rightarrow \mathbf{R}$ and $\sigma: \mathbf{R}^{+} \times \mathbf{R} \rightarrow \mathbf{R}$ are measurable, and $\{W(t), t \geq 0\}$ is the Wiener process with respect to the filtration $\left\{\mathcal{F}_{t}, t \geq 0\right\}$ defined on the probability space $(\Omega, \mathcal{F}, \mathrm{P})$.

First we list some conditions to be imposed on the coefficients of the equation. The conditions imposed on the nonrandom coefficients $b$ and $\sigma$ are as follows (these are the so-called Yamada conditions; see, for example, [1] and [4]):

(Y1) the functions $b$ and $\sigma$ are continuous with respect to all arguments;

(Y2) the linear growth:

$$
|b(t, x)|+|\sigma(t, x)| \leq C(1+|x|), \quad t \geq 0, x \in \mathbf{R}
$$

(Y3) the Lipschitz condition for $b$ :

$$
|b(t, x)-b(t, y)| \leq C|x-y|, \quad t \geq 0, x, y \in \mathbf{R}
$$

(Y4) there exists an increasing function $\rho: \mathbf{R}^{+} \rightarrow \mathbf{R}^{+}$such that $\int_{0+} \rho^{-2}(u) d u=+\infty$ and

$$
|\sigma(t, x)-\sigma(t, y)| \leq C \rho(|x-y|), \quad t \geq 0, x, y \in \mathbf{R} .
$$

Remark 2.1. A particular case of the function satisfying condition (Y4) is presented by

$$
\rho(x)=x^{\alpha}, \quad \frac{1}{2} \leq \alpha \leq 1 .
$$

Therefore (Y4) can be called the Hölder condition for $\sigma$. Below are some other examples of functions satisfying (Y4):

$$
\rho(x)=x^{1 / 2}(\ln 1 / x)^{1 / 2}, \quad \rho(x)=x^{1 / 2}(\ln 1 / x)^{1 / 2}(\ln \ln 1 / x)^{1 / 2},
$$

and so on.

Consider the notion of a strong solution of equation (1).

Definition 2.2. A pair $(X, W)$ is called a strong solution of equation (1) if $X$ is adapted to the flow $\left\{\overline{\mathcal{F}}_{t}^{W}\right\}$, that is, to the completed filtration generated by the process $W$.

Definition 2.3. We say that equation (11) has the property of pathwise uniqueness of a solution if

$$
\mathrm{P}(\forall t>0: X(t)=\tilde{X}(t))=1
$$

for all pairs of solutions $(X, W)$ and $(\tilde{X}, W)$ defined with respect to the same filtration. 
Definition 2.4. Let $T>0$. Let $\mathcal{L}_{2}^{T}(\mathcal{F}, \mathrm{P})$ be the space

$$
\mathcal{L}_{2}(\Omega \times[0, T], \mathcal{F} \otimes B([0, T]), \mathrm{P} \times \lambda)
$$

of stochastic processes satisfying the following condition:

$$
\int_{\Omega} \int_{0}^{T} X^{2}(t, \omega) \lambda(d t) \mathrm{P}(d \omega)<\infty .
$$

Theorem 2.5 (4]). If conditions (Y1)-(Y4) hold, then equation (11) has a unique strong solution belonging to the space $\mathcal{L}_{2}^{T}(\mathcal{F}, \mathrm{P})$ for all $T>0$.

Definition 2.6. Let $\tau_{n}$ be the first exit moment of the process $X(t)$ from the set $|x|<n$, that is,

$$
\tau_{n}=\inf \{t: X(t) \notin(-n, n)\} .
$$

Denote by $\tau=\lim _{n \rightarrow \infty} \tau_{n}$ the limit (finite or infinite) of the nondecreasing sequence $\tau_{n}$ as $n \rightarrow \infty$. The random variable $\tau$ is called the first exit moment of the trajectory from an arbitrary bounded domain or the entrance moment to infinity. A process $X(t)$ is called regular if

$$
\mathrm{P}\{\tau=\infty\}=1 .
$$

Remark 2.7. Theorem 2.5 implies that if Yamada's conditions hold, then a solution of equation (11) is a regular process with continuous trajectories.

\section{The Positivity of a SOlUtion FOR a STOCHASTIC DifFERENTIAL EQUATION WITH NONHOMOGENEOUS NON-LIPSCHITZ COEFFICIENTS}

Consider the following stochastic differential equation:

$$
X(t)=X_{0}+\int_{0}^{t} b(s, X(s)) d s+\int_{0}^{t} \sigma(s) \sqrt{|X(s)|} d W(s), \quad t \geq 0,
$$

where the initial value $X_{0}>0$ is nonrandom, the coefficients $b: \mathbf{R}^{+} \times \mathbf{R} \rightarrow \mathbf{R}$ and $\sigma: \mathbf{R}^{+} \rightarrow \mathbf{R}$ are measurable, and $\{W(t), t \geq 0\}$ is a Wiener process with respect to the filtration $\left\{\mathcal{F}_{t}, t \geq 0\right\}$ defined on the probability space $(\Omega, \mathcal{F}, \mathrm{P})$.

Assume that the coefficients of this equation satisfy Yamada's conditions (Y1)-(Y4) and that

$$
\inf _{t>0} \frac{1}{2} \sigma^{2}(t)=d>0 .
$$

Theorem 3.1. If all the above assumptions hold and additionally

$$
b(t, 0)>\frac{1}{2} \sigma^{2}(t)
$$

for all $t \geq 0$, then the process $X(t)$ does not vanish with probability one.

Remark 3.2. Recall that the condition $a b \geq \sigma^{2} / 2$ is necessary and sufficient that a process does not vanish in the homogeneous Cox-Ingersoll-Ross model described by the stochastic differential equation

$$
d r_{t}=a\left(b-r_{t}\right) d t+\sigma \sqrt{r_{t}} d W_{t}
$$

with the initial condition $r_{0}>0$. In our nonhomogeneous case of $b(t, x)=a(t)(b(t)-x)$ we obtain the condition $a(t) b(t)>\sigma^{2}(t) / 2$. 
Proof. Let $T>0$ be fixed. First we consider the case of $t \in[0, T]$ and prove that the process $X(t)$ does not vanish in this time interval. By the assumptions of the theorem, the continuity of the functions $b(t, x)$ and $\frac{1}{2} \sigma^{2}(t)$ (Yamada's condition (Y1)) imply that

$$
\begin{gathered}
\forall t>0 \quad \exists c(t)>0, x(t)>0: \quad \forall s \in(t-c(t), t+c(t)) \quad \forall x \in(0, x(t)) \\
b(s, x)>\frac{1}{2} \sigma^{2}(s) .
\end{gathered}
$$

Note that

$$
\bigcup_{t>0}(t-c(t), t+c(t))
$$

is an open covering of the compact set $[0, T]$. Thus one can find a finite subcovering

$$
\bigcup_{i=1}^{N}\left(t_{i}-c\left(t_{i}\right), t_{i}+c\left(t_{i}\right)\right) \text {. }
$$

Hence

$$
\begin{gathered}
\forall s \in(0, T) \quad \exists i, 1 \leq i \leq N: \quad s \in\left(t_{i}-c\left(t_{i}\right), t_{i}+c\left(t_{i}\right)\right) \text { and } \forall x \in\left(0, x\left(t_{i}\right)\right) \\
b(s, x)>\frac{1}{2} \sigma^{2}(s) .
\end{gathered}
$$

Put

$$
c:=\min _{1 \leq i \leq N} x\left(t_{i}\right)
$$

Then

$$
\forall t \in(0, T) \quad \forall x \in(0, c): \quad b(t, x)>\frac{1}{2} \sigma^{2}(t) .
$$

We define the function $V(x)$ by

$$
V(x)= \begin{cases}\ln x-\ln c+\frac{d}{a c} & \text { if } 0<x<c, \\ \frac{d}{a c} e^{a(x-c) / d} & \text { if } c \leq x<\infty,\end{cases}
$$

where $a$ is the constant defined in condition (Y2) as follows. If $x \in(c,+\infty)$, then condition (Y2) implies that there exists $K>0$ such that

$$
|b(t, x)| \leq K(1+x) .
$$

Put

Then we get

$$
a=K\left(1+\frac{1}{c}\right)
$$

$$
|b(t, x)| \leq a x, \quad \text { for all } x \in(c, \infty) .
$$

It is easy to see that the function $V(x)$ is continuously differentiable in $(0,+\infty)$ and that $V^{\prime}(x)$ is given by

$$
V^{\prime}(x)= \begin{cases}\frac{1}{x} & \text { if } 0<x<c, \\ \frac{1}{c} e^{a(x-c) / d} & \text { if } c \leq x<\infty .\end{cases}
$$

Consider the differential operator

$$
L=\frac{1}{2} \sigma^{2}(t) x \frac{\partial^{2}}{\partial x^{2}}+b(t, x) \frac{\partial}{\partial x} .
$$

Then, for $x \in(0, c)$,

$$
L V=\frac{1}{2} \sigma^{2}(t) x\left(-\frac{1}{x^{2}}\right)+b(t, x) \frac{1}{x}=\frac{1}{x}\left(b(t, x)-\frac{1}{2} \sigma^{2}(t)\right)>0
$$


by the choice of $c$. For $x \in(c,+\infty)$, we have

$$
\begin{aligned}
L V & =\frac{1}{2} \sigma^{2}(t) x \cdot \frac{a}{c d} e^{a(x-c) / d}+b(t, x) \cdot \frac{1}{c} e^{a(x-c) / d} \\
& \geq d x \cdot \frac{a}{c d} e^{a(x-c) / d}-a x \cdot \frac{1}{c} e^{a(x-c) / d}=0 .
\end{aligned}
$$

Thus $L V \geq 0$ for all $x>0, x \neq c$.

Let $0<\varepsilon<A$ be some fixed constant. Consider the random variable $\tau_{\varepsilon, A}$ defined by

$$
\tau_{\varepsilon, A}=\inf \{t: X(t)=\varepsilon \text { or } X(t)=A\} .
$$

We use the following generalization of the Itô formula proved in the paper [2]. Let a process $X=\left\{X_{t}, t \geq 0\right\}$ be a continuous semimartingale and let $v: \mathbf{R}_{+} \rightarrow \mathbf{R}_{+}$be a function of bounded variation. Put

$$
C=\left\{(t, x) \in \mathbf{R}_{+} \times[0, v(t)]\right\}
$$

and

$$
D=\left\{(t, x) \in \mathbf{R}_{+} \times[v(t),+\infty)\right\} .
$$

Assume that the function $F: \mathbf{R}_{+} \times \mathbf{R}_{+} \rightarrow \mathbf{R}$ is such that $F \in C^{1,2}(C)$ and $F \in C^{1,2}(D)$. Then

$$
\begin{aligned}
F\left(t, X_{t}\right)= & F\left(0, X_{0}\right)+\int_{0}^{t} \frac{1}{2}\left(F_{t}\left(s, X_{s}+\right)+F_{t}\left(s, X_{s}-\right)\right) d s \\
& +\int_{0}^{t} \frac{1}{2}\left(F_{x}\left(s, X_{s}+\right)+F_{x}\left(s, X_{s}-\right)\right) d X_{s} \\
& +\int_{0}^{t} F_{x x}\left(s, X_{s}\right) I\left(X_{s} \neq v(s)\right) d\langle X, X\rangle_{s} \\
& +\int_{0}^{t} \frac{1}{2}\left(F_{x}\left(s, X_{s}+\right)-F_{x}\left(s, X_{s}-\right)\right) I\left(X_{s}\right. \\
= & v(s)) d l_{s}^{v}(X),
\end{aligned}
$$

where

$$
l_{s}^{b}(X)=\mathrm{P}-\lim _{\varepsilon \downarrow 0} \frac{1}{2 \varepsilon} \int_{0}^{s} I\left(v(r)-\varepsilon<X_{r}<v(r)+\varepsilon\right) d\langle X, X\rangle_{r} .
$$

Since $V^{\prime}(x)$ is continuous in the interval $[\varepsilon,+\infty), V$ does not depend on $t$, and $v(s) \equiv c$, we obtain

$$
\begin{aligned}
V\left(X\left(\tau_{\varepsilon, A} \wedge T\right)\right)= & V\left(X_{0}\right)+\int_{0}^{\tau_{\varepsilon, A} \wedge T} V^{\prime}(X(s)) d X(s) \\
& +\frac{1}{2} \int_{0}^{\tau_{\varepsilon, A} \wedge T} V^{\prime \prime}(X(s)) I(X(s) \neq c) d\langle X, X\rangle_{s} \\
= & V\left(X_{0}\right)+\int_{0}^{\tau_{\varepsilon, A} \wedge T} V^{\prime}(X(s)) b(s, X(s)) d s \\
& +\int_{0}^{\tau_{\varepsilon}, A} V^{\prime}(X(s)) \sigma(s, X(s)) d W(s) \\
& +\frac{1}{2} \int_{0}^{\tau_{\varepsilon, A} \wedge T} V^{\prime \prime}(X(s)) I(X(s) \neq c) \sigma^{2}(s) X(s) d s
\end{aligned}
$$

Here we used the property that

$$
d\langle X, X\rangle_{s}=\sigma^{2}(s) X(s) d s .
$$

Since the function $b(t, x)$ is bounded in $x \in[\varepsilon, A]$ and separated from 0 and $\infty$ and since $\sigma(t, x)=\sigma(t) \sqrt{x}$ is a uniformly Hölder function with respect to $x$, we deduce from [3] that 
$\mathrm{P}\left(X(s)=c, \tau_{\varepsilon, A}<s\right)=0$. Indeed, one can extend the coefficients $b(t, x)$ and $\sigma(t) \sqrt{x}$ beyond the interval $[\varepsilon, A]$ with respect to the argument $x$ in such a way that all the above conditions hold. Let $Y$ be a solution of the corresponding equation. Then

$$
\mathrm{P}\left(X(s)=c, \tau_{\varepsilon, A}<s\right) \leq \mathrm{P}(Y(s)=c)=0,
$$

since $Y(s)$ has density with respect to Lebesgue measure (see [3]).

Further,

$$
\begin{aligned}
0 & \leq \mid \mathrm{E} \int_{0}^{\tau_{\varepsilon, A} \wedge T}\left(V^{\prime}(X(s)) b(s, X(s)) I(X(s)=c) d s \mid\right. \\
& \leq\left|V^{\prime}(c)\right| \max _{s \in[0, T]}|b(s, c)| \int_{0}^{T} \mathrm{P}\left(X(s)=c, \tau_{\varepsilon, A}<s\right) d s=0,
\end{aligned}
$$

whence $\mathrm{E} \int_{0}^{\tau_{\varepsilon, A} \wedge T}\left(V^{\prime}(X(s)) b(s, X(s)) I(X(s)=c) d s=0\right.$.

Considering the mathematical expectation of both sides of the equality

$$
\begin{aligned}
V\left(X\left(\tau_{\varepsilon, A} \wedge T\right)\right)= & V\left(X_{0}\right)+\int_{0}^{\tau_{\varepsilon, A} \wedge T} V^{\prime}(X(s)) b(s, X(s)) d s \\
& +\int_{0}^{\tau_{\varepsilon, A} \wedge T} V^{\prime}(X(s)) \sigma(s, X(s)) d W(s) \\
& +\frac{1}{2} \int_{0}^{\tau_{\varepsilon, A} \wedge T} V^{\prime \prime}(X(s)) I(X(s) \neq c) \sigma^{2}(s) X(s) d s,
\end{aligned}
$$

we get

$$
\begin{aligned}
\mathrm{E}\left(V\left(X\left(\tau_{\varepsilon, A} \wedge T\right)\right)\right) \\
=V\left(X_{0}\right)+\mathrm{E} \int_{0}^{\tau_{\varepsilon, A} \wedge T} V^{\prime}(X(s)) b(s, X(s)) d s \\
\quad+\mathrm{E} \int_{0}^{\tau_{\varepsilon}, A \wedge T} \frac{1}{2} V^{\prime \prime}(X(s)) I(X(s) \neq c) \sigma^{2}(s) X(s) d s \\
=V\left(X_{0}\right)+\mathrm{E} \int_{0}^{\tau_{\varepsilon, A} \wedge T} V^{\prime}(X(s)) b(s, X(s)) I(X(s)=c) d s \\
\quad+\mathrm{E} \int_{0}^{\tau_{\varepsilon, A} \wedge T}\left(V^{\prime}(X(s)) b(s, X(s))+\frac{1}{2} V^{\prime \prime}(X(s)) \sigma^{2}(s) X(s)\right) I(X(s) \neq c) d s \\
=V\left(X_{0}\right)+\mathrm{E} \int_{0}^{\tau_{\varepsilon, A} \wedge T}\left(V^{\prime}(X(s)) b(s, X(s))+\frac{1}{2} V^{\prime \prime}(X(s)) \sigma^{2}(s) X(s)\right) I(X(s) \neq c) d s .
\end{aligned}
$$

Since the function $V(x)$ is bounded from above in the interval $[0, A]$,

$$
\mathrm{E}\left(V\left(X\left(\tau_{\varepsilon, A} \wedge T\right)\right)\right) \leq \max _{x \in[0, A]} V(x)+V(\varepsilon) \mathrm{P}\left(\tau=\tau_{\varepsilon}\right),
$$

where $\tau_{A}$ and $\tau_{\varepsilon}$ are the first hitting moments for the boundaries $A$ and $\varepsilon$, respectively, and where $\tau=\tau_{A} \wedge \tau_{\varepsilon} \wedge T$.

Thus

$$
\begin{aligned}
\max _{x \in[0, A]} V(x)+V(\varepsilon) \mathrm{P}\left(\tau=\tau_{\varepsilon}\right) \\
\geq V\left(X_{0}\right)+\mathrm{E} \int_{0}^{\tau_{\varepsilon, A} \wedge T}\left(V^{\prime}(X(s)) b(s, X(s)) I(X(s)=c) d s\right. \\
\quad+\mathrm{E}\left(\int_{0}^{\tau_{\varepsilon, A} \wedge T}\left(V^{\prime}(X(s)) b(s, X(s))+\frac{1}{2} V^{\prime \prime}(X(s)) \sigma^{2}(s) X(s)\right) I(X(s) \neq c) d s\right) .
\end{aligned}
$$


Then

$$
\begin{aligned}
& \max _{x \in[0, A]} V(x)+V(\varepsilon) \mathrm{P}\left(\tau=\tau_{\varepsilon}\right) \\
& \geq V\left(X_{0}\right) \\
& +\mathrm{E}\left(\int_{0}^{\tau_{\varepsilon, A} \wedge T}\left(V^{\prime}(X(s)) b(s, X(s))+\frac{1}{2} V^{\prime \prime}(X(s)) \sigma^{2}(s) X(s)\right) I(X(s) \neq c) d s\right) \\
& =V\left(X_{0}\right)+\mathrm{E}\left(\int_{0}^{\tau_{\varepsilon, A} \wedge T} L V(X(s)) I(X(s) \neq c) d s\right) \geq V\left(X_{0}\right) .
\end{aligned}
$$

Letting $\varepsilon \rightarrow 0$ and taking into account that

$$
V(\varepsilon) \rightarrow-\infty, \quad \varepsilon \rightarrow 0,
$$

we prove that the left hand side of the inequality approaches $-\infty$, while the right hand side is constant. This leads to a contradiction, and we conclude that

$$
\mathrm{P}\left(\tau=\tau_{0}\right)=0 .
$$

Therefore equality (3) holds for all fixed $A$ and $T$. Passing to the limit as $A$ and $T$ tend to $+\infty$, we complete the proof of the theorem.

Remark 3.3. Since the initial value of the process $X(t), t \geq 0$, is positive and since its trajectories do not vanish with probability one (and thus are positive), one can omit the sign of the absolute value in equation (2).

\section{A Limit theorem for EQUations With NONHOMOGENEOUS COEFFICIENTS} AND NON-LIPSCHITZ DIFFUSION

Consider a sequence of stochastic differential equations

$$
X_{n}(t)=X_{n}(0)+\int_{0}^{t} b_{n}\left(s, X_{n}(s)\right) d s+\int_{0}^{t} \sigma_{n}\left(s, X_{n}(s)\right) d W(s), \quad n \geq 0
$$

with nonrandom initial conditions $X_{n}(0)$. Assume that the coefficients of these equations satisfy conditions analogous to (Y1)-(Y4); namely,

$\left(\mathrm{Y} 1_{n}\right) b_{n}$ and $\sigma_{n}$ are continuous with respect to all arguments;

$\left(\mathrm{Y} 2_{n}\right)$ linear growth:

$$
\left|\sigma_{n}(t, x)\right|+\left|b_{n}(t, x)\right| \leq L(1+|x|), \quad t \geq 0, x \in \mathbf{R} ;
$$

$\left(\mathrm{Y} 3_{n}\right)$ Lipschitz condition for $b_{n}$ :

$$
\left|b_{n}(t, x)-b_{n}(t, y)\right| \leq L|x-y|, \quad t \geq 0, x, y \in \mathbf{R} .
$$

$\left(\mathrm{Y} 4_{n}\right)$ there exists an increasing function $\rho_{n}: \mathbf{R}^{+} \rightarrow \mathbf{R}^{+}$such that $\int_{0+} \rho_{n}^{-2}(u) d u=\infty$ and

$$
\left|\sigma_{n}(t, x)-\sigma_{n}(t, y)\right| \leq \rho_{n}(|x-y|), \quad t \geq 0, x, y \in \mathbf{R} .
$$

We also assume that, as $n \rightarrow \infty$,

$$
X_{n}(0) \rightarrow X_{0}(0), \quad b_{n}(t, x) \rightarrow b_{0}(t, x), \quad \sigma_{n}(t, x) \rightarrow \sigma_{0}(t, x)
$$

for $t \geq 0$ and $x \in \mathbf{R}$.

Theorem 4.1. If conditions (50)-(6) hold, then

$$
\mathrm{E}\left[\left|X_{n}(t)-X_{0}(t)\right|\right] \rightarrow 0, \quad n \rightarrow \infty,
$$

uniformly in any finite interval. 
Proof. The existence and uniqueness of a solution of equation (4) follows from Theorem 2.5. According to this theorem, we also have $\mathrm{E}\left[X_{n}^{2}(t)\right]<\infty$. Moreover, the second moments are uniformly bounded on finite intervals in view of the Gronwall inequality. Put

$$
\widetilde{X}_{n}(t)=X_{n}(0)+\int_{0}^{t} b_{n}\left(s, X_{0}(s)\right) d s+\int_{0}^{t} \sigma_{n}\left(s, X_{0}(s)\right) d W(s)
$$

Then

$$
\begin{aligned}
& \mathrm{E}\left[\left(\widetilde{X}_{n}(t)-X_{0}(t)\right)^{2}\right] \\
& \begin{aligned}
\leq C\left[\left(X_{n}(0)-X_{0}(0)\right)^{2}+\right. & t \int_{0}^{t} \mathrm{E}\left[\left(b_{n}\left(s, X_{0}(s)\right)-b_{0}\left(s, X_{0}(s)\right)\right)^{2}\right] d s \\
& \left.+\int_{0}^{t} \mathrm{E}\left[\left(\sigma_{n}\left(s, X_{0}(s)\right)-\sigma_{0}\left(s, X_{0}(s)\right)\right)^{2}\right] d s\right] .
\end{aligned}
\end{aligned}
$$

Conditions (6) and $\mathrm{E}\left[X_{0}^{2}(t)\right] \leq C$ imply the dominated convergence property, whence

$$
\mathrm{E}\left[\left(\widetilde{X}_{n}(t)-X_{0}(t)\right)^{2}\right] \rightarrow 0, \quad n \rightarrow \infty
$$

Moreover, the convergence is uniform in bounded intervals, since the right hand side of inequality (8) increases in $t$. Hence

$$
\sup _{t \in[0, T]} \mathrm{E}\left[\left|\widetilde{X}_{n}(t)-X_{0}(t)\right|\right] \rightarrow 0, \quad n \rightarrow \infty
$$

for $T>0$.

Now we follow the Yamada method [5]. Let the numbers $1>a_{1}^{n}>a_{2}^{n}>\cdots>0$ be determined by the equalities

$$
\begin{gathered}
\int_{a_{1}^{n}}^{1} \rho_{n}^{-2}(u) d u=1, \quad \int_{a_{2}^{n}}^{a_{1}^{n}} \rho_{n}^{-2}(u) d u=2, \quad \int_{a_{3}^{n}}^{a_{2}^{n}} \rho_{n}^{-2}(u) d u=3, \\
\int_{a_{m}^{n}}^{a_{m-1}^{n}} \rho_{n}^{-2}(u) d u=m .
\end{gathered}
$$

For every $n \geq 1$, we construct even functions $\varphi_{m}^{n}(x): \mathbf{R} \rightarrow[0,1]$ such that $\varphi_{m}^{n} \in C^{\infty}(\mathbf{R})$, $\varphi_{m}^{n}(0)=0,\left(\varphi_{m}^{n}\right)^{\prime}(x)=0, x \in\left[0, a_{m}^{n}\right),\left(\varphi_{m}^{n}\right)^{\prime}(x)=1, x>a_{m-1}^{n}$, and

$$
0 \leq\left(\varphi_{m}^{n}\right)^{\prime \prime}(x) \leq \frac{2}{m \rho_{n}^{2}(x)}, \quad x \in\left[a_{m}^{n}, a_{m-1}^{n}\right] .
$$

Such functions exist, indeed, since one can choose, for example,

$$
\psi_{m}^{n}(x) \in C^{\infty}([0, \infty))
$$

with $\operatorname{supp} \psi_{m}^{n} \subset\left[a_{m}^{n}, a_{m-1}^{n}\right], 0 \leq \psi_{m}^{n}(x) \leq 2 /\left(m \rho_{n}^{2}(x)\right), \int_{0}^{\infty} \psi_{m}^{n}(u) d u=1$ and set

$$
\varphi_{m}^{n}(x)=\int_{0}^{|x|} d y \int_{0}^{y} \psi_{m}^{n}(u) d u, \quad x \in \mathbf{R} .
$$


It is clear that $\varphi_{m}^{n}(x) \uparrow|x|$ as $m \rightarrow \infty$, since $\int_{0}^{y} \psi_{m}^{n}(u) d u \rightarrow 1$ as $m \rightarrow \infty$. According to the Itô formula,

$$
\begin{aligned}
\mathrm{E}\left[\varphi_{m}^{n}\right. & \left.\left(X_{n}(t)-\widetilde{X}_{n}(t)\right)\right] \\
= & \int_{0}^{t} \mathrm{E}\left[\left(\varphi_{m}^{n}\right)^{\prime}\left(X_{n}(s)-X_{0}(s)\right)\left[b_{n}\left(X_{n}(s)\right)-b_{n}\left(X_{0}(s)\right)\right]\right] d s \\
& +\frac{1}{2} \int_{0}^{t} \mathrm{E}\left[\left(\varphi_{m}^{n}\right)^{\prime \prime}\left(X_{n}(s)-X_{0}(s)\right)\left[\sigma_{n}\left(X_{n}(s)\right)-\sigma_{n}\left(X_{0}(s)\right)\right]^{2}\right] d s \\
\leq & C \int_{0}^{t} \mathrm{E}\left[\left|X_{n}(s)-X_{0}(s)\right|\right] d s \\
& +\frac{1}{m} \int_{0}^{t} \mathrm{E}\left[\rho_{n}^{-2}\left(\left|X_{n}(s)-X_{0}(s)\right|\right) \rho_{n}^{2}\left(\left|X_{n}(s)-X_{0}(s)\right|\right)\right] d s \\
\leq & \frac{t}{m}+C \int_{0}^{t} \mathrm{E}\left[\left|X_{n}(s)-X_{0}(s)\right|\right] d s .
\end{aligned}
$$

Letting $m \rightarrow \infty$ and using Fatou's lemma, we get

$$
\mathrm{E}\left[\left|X_{n}(t)-\widetilde{X}_{n}(t)\right|\right] \leq C \int_{0}^{t} \mathrm{E}\left[\left|X_{n}(s)-X_{0}(s)\right|\right] d s
$$

Put

$$
Z(t)=\sup _{t \in[0, T]} \mathrm{E}\left[\left|X_{n}(t)-X_{0}(t)\right|\right]
$$

Then

$$
Z(T) \leq \sup _{t \in[0, T]} \mathrm{E}\left|\widetilde{X}_{n}(t)-X_{0}(t)\right|+C \int_{0}^{T} Z(s) d s
$$

whence

$$
Z(T) \leq C \sup _{t \in[0, T]} \mathrm{E}\left|\widetilde{X}_{n}(t)-X_{0}(t)\right| \rightarrow 0, \quad n \rightarrow \infty,
$$

by the Gronwall inequality.

\section{CONCluding REMARKS}

We found conditions imposed on the nonhomogeneous coefficients of a stochastic differential equation with the diffusion $\sigma(t, x)=\sigma(t) \sqrt{x}$ ensuring that a solution does not vanish over a finite time. The result obtained in the paper can be applied to the nonhomogeneous Cox-Ingersoll-Ross model by using a condition on the positivity of a solution, similar to that used in the homogeneous case. We also proved a limit theorem on the continuous dependence on the parameter $n$ in the space $L_{1}(\mathrm{P})$ of solutions of a sequence

of stochastic differential equations with nonhomogeneous coefficients and non-Lipschitz diffusion.

\section{BIBLIOGRAPHY}

1. N. Ikeda and S. Watanabe, Stochastic Differential Equations and Diffusion Processes, NorthHolland Publishing Co. and Kodansha, Ltd., Amsterdam-New York and Tokyo, 1981. MR637061 (84b:60080)

2. G. Peskir, A change-of-variable formula with local time on curves, J. Theoret. Probab. 18 (2005), no. 3, 499-535. MR2167640(2006k:60096)

3. D. W. Stroock and S. R. S. Varadhan, Diffusion processes with continuous coefficients I, Comm. Pure Appl. Math. 22 (1969), no. 3, 345-400. MR0253426 (40:6641) 
4. T. Yamada, Sur l'approximation des solutions d'équations différentielles stochastiques, Z. Wahrscheinlichkeitstheorie und Verw. Gebiete 36 (1976), 153-164. MR0413269 (54:1386)

5. T. Yamada and S. Watanabe, On the uniqueness of solutions of stochastic differential equations, J. Math. Kyoto Univ. 11 (1971), 155-167. MR.0278420 (43:4150)

Department of Probability Theory and Mathematical Statistics, Faculty for Mechanics and Mathematics, National Taras Shevchenko University, Academician Glushkov Avenue 6 , KYIV 03127, UKRAINE

E-mail address: myus@univ.kiev.ua

Department of Probability Theory and Mathematical Statistics, Faculty for Mechanics and Mathematics, National Taras Shevchenko University, Academician Glushkov Avenue 6 , KYIV 03127, UKRAINE

E-mail address: revan1988@gmail.com

Department of Probability Theory and Mathematical Statistics, Faculty for Mechanics and Mathematics, National Taras Shevchenko University, Academician Glushkov Avenue 6 , KYIV 03127, UKRAINE

E-mail address: zhora@univ.kiev.ua

Received 12/MAR/2008

Translated by S. KVASKO 